advantage when it come physical and mental health, economic, and social outcomes; delayed or withheld treatment only further disadvantages an already vulnerable population. Understanding who is most vulnerable in this situation is one step towards improving outcomes for system involved youth.

Sources of Support: FUNDING NUMBER: A93-3-13-4F-C0-0981.

98.

\section{WELL-BEING AND YOUTHS: IS SLEEPING THE KEY?}

Yara Barrense-Dias, $M A^{1}$, André Berchtold, $P h D^{2}$, Christina Akre, $P h D^{1}$, Joan-Carles Suris, $M D, M P H, P h D^{1}$

${ }^{1}$ Lausanne University Hospital; ${ }^{2}$ Lausanne University.

Purpose: To assess the elements that could affect changes in emotional well-being among youths.

Methods: 1606 youths (mean age 16.2 at baseline [15-24], 45.8\% females) from eleven post-mandatory schools in the Canton of Fribourg, Switzerland, participated in the first two waves (winter 2016 (T0) and winter 2017 (T1)) of a Swiss longitudinal study on lifestyle (GenerationFRee). Using the WHO-Five Well-Being Index with a cut-off at 13/25 indicating a poor emotional well-being $(\mathrm{WB})$, participants were divided into four groups according to the evolution of their WB between T0 and T1: (1) good at T0 and T1 (GT0T1: 69.3\%), (2) good at T0 and poor at T1 (GTOPT1: 10.5\%), (3) poor at T0 and good at T1 (PT0GT1: 9.1\%), (4) poor at T0 and T1 (PT0T1: 11.1\%). Groups were compared on socio-demographic, family, academic and health data (perceived health status, disordered eating, somatic complaints) at T0 and T1. Significant $(\mathrm{p}<.05)$ variables at the bivariate level were included in a multinomial regression analysis using GTOT1 as the reference category. Data are presented as relative risks ratios (RRR).

Results: At the bivariate level, compared to GTOT1, participants in the other groups were more likely to be females and older, to report poorer relationships with parents, a poor perceived health status, disordered eating and somatic complaints. Differences were also found for socioeconomic status and academic track. No significant difference was found in terms of living in an urban or rural area and having an intact family. In the multivariate analysis, compared to the GTOT1, GTOPT1 participants were more likely to be students (vs. apprentices) (RRR: 1.75) and to report at T1 a poorer relationship with their father (RRR: .87), a poorer health status (RRR: 4.17) and more back pain (RRR: 2.10) and sleep problems (RRR: 3.03). Those in the PTOGT1 group were more likely to be older (RRR: 1.21) and students (RRR: 2.22), and to report at T0 a poorer relationship with their mother (RRR: .81 ), disordered eating (RRR: 1.83) and sleep problems (RRR: 2.44). Finally, those in the PTOT1 group were more likely to report a poor relationship with their father at T1 (RRR: .85), a poorer health status both at T0 and T1 (RRR: 3.87 / 4.46), disordered eating at T1 (RRR: 1.98), stomach pain at T1 (RRR: 1.94) and sleep problems both at T0 and T1 (RRR: 3.24 / 3.80).

Conclusions: Young people's well-being is a complex issue that can be affected by several factors and can also negatively impact their lives. In this study, sleep problems were common to all the groups reporting poor well-being at some point. Sleep improvement or deterioration directly affected well-being in the same way. Sleep complaints could be used as an indirect way to screen for emotional wellbeing.

Sources of Support: The Programme Intercantonal de Lutte contre la Dépendance au Jeu (PILDJ) and the canton of Fribourg.
99.

\section{EXAMINING ITEM CHARACTERISTICS AND DIF OF THE MDS FOR YOUNG ADOLESCENTS: IMPLICA- TIONS FOR COMMUNITY MENTAL HEALTH RESEARCH}

Andrea Johnson, PhD (C), Mauricio Coronel Villalobos, Sneha Shankar, Sheila Marshal, $P h D$

The University of British Columbia.

Purpose: Depression in adolescents is a common but often underrepresented global mental health problem (Thapar, Collishaw, Pine, \& Thapar, 2012). The prevalence of depression rises significantly at the onset of adolescence making pre and early adolescence difficult periods to detect symptoms as some may be obscure. Therefore, there are difficulties detecting symptoms when conducting communitybased mental health research with pre and early adolescents. Few brief measurement tools exist for assessing depressive symptoms. The Modified Depression Scale (MDS) (Bosworth, Espelage, \& Simon, 1996) is a self-administered 6-item measure that assesses the frequency of depressive symptoms adolescents might be experiencing and therefore is useful in community-based health research. Although reliability and validity evidence has been reported for middle to late adolescents attending high school (Dunn, Johnson, \& Green, 2011), it is unknown if the items in the MDS accurately detect depressive symptoms among pre and early adolescents. This investigation sought to examine whether the MDS is an appropriate survey assessment of depressive symptoms among this population by testing the function of each item as well as the relationships among items.

Methods: A survey was administered during class periods to students in grades 6 to $8(\mathrm{~N}=325 ; 55.4 \%$ girls $)$ aged 10 to 14 years (Mage =12.06; $\mathrm{SD}=.87$ ) and attending a single school district in central British Columbia, Canada. Most participants were born in Canada (91.7\%) and spoke English at home (99.4\%). Students completed the six items from the MDS as part of a larger study of development during early adolescence. The items assess sadness, irritability, hopelessness, change in appetite, sleep problems, and concentration difficulties. Responses to items assess frequency of symptoms in the past month using a five-point, Likert-type scale ranging from (1) never to (5) always. The software flexMIRT 3.03 (Cai, 2013) was used to examine item responses. A two-Parameter Logistic (2PL) model was used to estimate model fit, item and test information values from -2.8 to 2.8 theta, and examine DIF between boys and girls.

Results: Items 1 (Sadness), 3 (hopelessness) and 5 (sleep problems) presented acceptable fit, however, the overall model presented poor fit $(\mathrm{p}<.01, \mathrm{AIC}=4840.27$, BIC $=4953.78, \mathrm{RMSEA}=.13)$. Item information ranged from .01 to 1.20 ; test information ranged from 1.90 to 5.18. Items were most informative at higher levels of depressive symptoms. Analysis by gender shows that responses between boys and girls for item 1 presented overall DIF $(p=.03)$. When examined in detail, there is no difference in slope (discrimination), but the intercept presents a gender effect $(p=.02)$, where boys were more likely to endorse a lower value of sadness.

Conclusions: Although not presenting an optimal fit, which may be due to the expected skewed distributions of items, this analysis has demonstrated that the MDS is an appropriate survey assessment of depressive symptoms in pre and early adolescents. Although there is a gender effect on the intercept of the sadness item, the overall model works equally with boys and girls.

Sources of Support: This investigation is part of a larger SSHERC funded study on adolescent development. 